\title{
Infinitely Many Periodic Solutions of Duffing Equations with Singularities via Time Map
}

\author{
Tiantian Ma and Zaihong Wang \\ School of Mathematical Sciences, Capital Normal University, Beijing 100048, China \\ Correspondence should be addressed to Zaihong Wang; zhwang@cnu.edu.cn
}

Received 24 December 2013; Accepted 6 February 2014; Published 16 April 2014

Academic Editor: Shuangjie Peng

Copyright (C) 2014 T. Ma and Z. Wang. This is an open access article distributed under the Creative Commons Attribution License, which permits unrestricted use, distribution, and reproduction in any medium, provided the original work is properly cited.

We study the periodic solutions of Duffing equations with singularities $x^{\prime \prime}+g(x)=p(t)$. By using Poincaré-Birkhoff twist theorem, we prove that the given equation possesses infinitely many positive periodic solutions provided that $g$ satisfies the singular condition and the time map related to autonomous system $x^{\prime \prime}+g(x)=0$ tends to zero.

\section{Introduction}

In this paper, we are concerned with the periodic solutions of singular Duffing equations:

$$
x^{\prime \prime}+g(x)=p(t),
$$

where $g:(0,+\infty) \rightarrow \mathbf{R}$ is locally Lipschitz continuous and has a singularity at the origin and $p: \mathbf{R} \rightarrow \mathbf{R}$ is continuous and periodic, whose least period is $2 \pi$.

The periodic problem of equations with singularities has been widely studied lately because of their background in applied sciences [1-15]. For example, the oscillation problem of a spherical thick shell made of an elastic material can also be modeled by this kind of equations [1].

The opening work on the existence of periodic solutions of ordinary differential equations with singularities was done by Lazer and Solimini [2], in which the equations

$$
x^{\prime \prime}-\frac{1}{x^{\nu}}=p(t)
$$

were studied. It was proved in [2] that if $v \geq 1$, then (2) has at least one positive $2 \pi$-periodic solution if and only if

$$
\int_{0}^{2 \pi} p(t) d t<0
$$

Meanwhile, if $0<v<1$, then they constructed a periodic function $p(t)$ with negative mean value such that (2) does not have any $2 \pi$-periodic solution.
It is well known that time map plays an important role in studying the existence and multiplicity of periodic solutions of Duffing equations without singularities. In case when $g$ has a singularity, we can also use time map to deal with the periodic solutions of (1) (see [4] and the related references therein).

Assume that $g$ satisfies

$$
\lim _{x \rightarrow 0^{+}} g(x)=-\infty
$$

and the primitive function $G$ of $g$ satisfies

$$
\lim _{x \rightarrow 0^{+}} G(x)=+\infty, \quad\left(G(x)=\int_{1}^{x} g(s) d s\right) .
$$

Moreover, the following condition holds:

$$
\lim _{x \rightarrow+\infty} g(x)=+\infty \text {. }
$$

Let us define

$$
\tau(c)=\int_{1}^{c} \frac{d s}{\sqrt{G(c)-G(s)}} .
$$

The map $\tau$ is usually called time map, which is continuous for $c$ large enough. We shall deal with the multiplicity of periodic solutions of (1) by means of asymptotic property of the time map $\tau$. Assume that the time map satisfies

$$
\lim _{c \rightarrow+\infty} \tau(c)=0 .
$$


It is easy to check that if $g$ satisfies superlinear condition

$$
\lim _{x \rightarrow+\infty} \frac{g(x)}{x}=+\infty
$$

then condition $(\tau)$ is satisfied. However, the converse is not true. In fact, we can find functions $g$, which do not satisfy (5). But the corresponding time maps satisfy the condition $(\tau)$. For example, let us define

$$
g(x)= \begin{cases}3-\frac{1}{x}, & 0<x \leq 1 \\ x+(x-1)^{3} & \\ +(x-1)^{3} \sin (x-1)^{4}, & x \geq 1 .\end{cases}
$$

Obviously, conditions $\left(h_{i}\right)(i=1,2,3)$ hold and condition (5) does not hold. Next, we will show that condition $(\tau)$ is satisfied. In case when $x \geq 1$, we have

$$
\begin{aligned}
G(x) & =\int_{1}^{x}\left(s+(s-1)^{3}+(s-1)^{3} \sin (s-1)^{4}\right) d s \\
& =\frac{1}{2} x^{2}+\frac{1}{4}(x-1)^{4}-\frac{1}{4} \cos (x-1)^{4}-\frac{1}{4} .
\end{aligned}
$$

Therefore, we have

$$
\begin{gathered}
\lim _{c \rightarrow+\infty} \tau(c) \\
=\lim _{c \rightarrow+\infty} \int_{1}^{c}(d s) \\
\quad \times\left(\frac{1}{2}\left(c^{2}-s^{2}\right)+\frac{1}{4}\left((c-1)^{4}-(s-1)^{4}\right)\right. \\
\left.\quad+\frac{1}{4}\left(\cos (s-1)^{4}-\cos (c-1)^{4}\right)\right)^{-1 / 2} \\
=\lim _{c \rightarrow+\infty} \frac{1}{c} \int_{1 / c}^{1}(d t) \\
\quad \times\left(\frac{1}{2 c^{2}}\left(1-t^{2}\right)+\frac{1}{4 c^{4}}\left((c-1)^{4}-(c t-1)^{4}\right)\right. \\
\left.\quad+\frac{1}{4 c^{4}}\left(\cos (c t-1)^{4}-\cos (c-1)^{4}\right)\right)^{-1 / 2}
\end{gathered} .
$$

Since

$$
\begin{aligned}
& \lim _{c \rightarrow+\infty} \int_{1 / c}^{1}(d t) \times\left(\frac{1}{2 c^{2}}\left(1-t^{2}\right)+\frac{1}{4 c^{4}}\left((c-1)^{4}-(c t-1)^{4}\right)\right. \\
&\left.\quad+\frac{1}{4 c^{4}}\left(\cos (c t-1)^{4}-\cos (c-1)^{4}\right)\right)^{-1 / 2}
\end{aligned}
$$

we get

$$
\lim _{c \rightarrow+\infty} \tau(c)=0
$$

When the conditions $\left(h_{1}\right),\left(h_{2}\right)$, and (5) hold, it was proved in [6] that (1) has infinitely many periodic solutions.
In the present paper, we will deal with the multiplicity of periodic solutions of (1) under the conditions $\left(h_{1}\right),\left(h_{2}\right),\left(h_{3}\right)$, and $(\tau)$. Obviously, the conditions $\left(h_{3}\right)$ and $(\tau)$ generalize the condition (5). Since (5) does not hold, the estimating method in [6] is invalid. By taking some new estimating skills, we obtain the following results.

Theorem 1. Assume that conditions $\left(h_{i}\right)(i=1,2,3)$ and $(\tau)$ hold. Then (1) has infinitely many positive harmonic solutions $\left\{x_{j}(t)\right\}$ satisfying

$$
\begin{gathered}
\lim _{j \rightarrow \infty}\left(\min _{0 \leq t \leq 2 \pi}\left(x_{j}(t)+\left|x_{j}^{\prime}(t)\right|\right)\right)=0, \\
\lim _{j \rightarrow \infty}\left(\max _{0 \leq t \leq 2 \pi}\left(x_{j}(t)+\left|x_{j}^{\prime}(t)\right|\right)\right)=+\infty .
\end{gathered}
$$

Theorem 2. Assume that conditions $\left(h_{i}\right)(i=1,2,3)$ and $(\tau)$ hold. Then for any integer $m \geq 2$, (1) has infinitely many positive $m$-order subharmonic solutions $\left\{x_{j}(t)\right\}$ satisfying

$$
\begin{gathered}
\lim _{j \rightarrow \infty}\left(\min _{0 \leq t \leq 2 m \pi}\left(x_{j}(t)+\left|x_{j}^{\prime}(t)\right|\right)\right)=0, \\
\lim _{j \rightarrow \infty}\left(\max _{0 \leq t \leq 2 m \pi}\left(x_{j}(t)+\left|x_{j}^{\prime}(t)\right|\right)\right)=+\infty .
\end{gathered}
$$

Remark 3. In the following, for convenience and brevity, we move the singular point 0 to the point -1 . In fact, we can take a transformation $x=u+1$ to achieve this aim. We will consider singular equations as follows:

$$
x^{\prime \prime}+g(x)=p(t)
$$

where $g:(-1,+\infty) \rightarrow \mathbf{R}$ is continuous and has a singularity at $x=-1$. We now assume that the following conditions hold:

$$
\begin{aligned}
& \lim _{x \rightarrow-1^{+}} g(x)=-\infty, \\
& \lim _{x \rightarrow-1^{+}} G(x)=+\infty .
\end{aligned}
$$

Next, we will deal with the existence and multiplicity of periodic solutions of $\left(1^{\prime}\right)$ under conditions $\left(h_{1}^{\prime}\right),\left(h_{2}^{\prime}\right),\left(h_{3}\right)$, and $(\tau)$.

\section{Basic Lemmas}

In this section, we will perform some phase-plane analyses for $\left(1^{\prime}\right)$ when conditions $\left(h_{1}^{\prime}\right),\left(h_{2}^{\prime}\right)$, and $\left(h_{3}\right)$ hold. Consider the equivalent system of $\left(1^{\prime}\right)$ :

$$
x^{\prime}=y, \quad y^{\prime}=-g(x)+p(t) .
$$

Let $(x(t), y(t))=\left(x\left(t, x_{0}, y_{0}\right), y\left(t, x_{0}, y_{0}\right)\right)$ be the solution of (13) through the initial point:

$$
x\left(0, x_{0}, y_{0}\right)=x_{0}, \quad y\left(0, x_{0}, y_{0}\right)=y_{0} .
$$

Lemma 4. Assume that conditions $\left(h_{2}^{\prime}\right)$ and $\left(h_{3}\right)$ hold. Then every solution $(x(t), y(t))$ of system (13) exists uniquely on the whole t-axis. 
Proof. Define a potential function

$$
V(x, y)=\frac{1}{2} y^{2}+G(x) .
$$

Set

$$
V(t)=\frac{1}{2} y^{2}(t)+G(x(t))
$$

Then we have

$$
V^{\prime}(t)=p(t) y(t) \leq M|y(t)|
$$

where $M=\max \{|p(t)|: t \in \mathbf{R}\}$. From $\left(h_{2}^{\prime}\right)$ and $\left(h_{3}\right)$ we know that there exists a constant $M^{\prime}>0$ such that

$$
G(x)+M^{\prime} \geq 0, \quad x \in(-1,+\infty) .
$$

From (17) and (18) we get

$$
V^{\prime}(t) \leq M|y(t)|+G(x(t))+M^{\prime} \leq V(t)+M^{\prime \prime},
$$

where $M^{\prime \prime}=M^{\prime}+M^{2} / 2$. Then, for any finite $T>0$, we have

$$
V(t) \leq V(0) e^{T}+M^{\prime \prime}\left(e^{T}-1\right), \quad t \in[0, T) .
$$

Therefore, $(x(t), y(t))$ is bounded for $t \in[0, T)$. Furthermore, $(x(t), y(t))$ exists on the interval $[0,+\infty)$. Similarly, we can prove that $(x(t), y(t))$ exists on the interval $(-\infty, 0]$. The uniqueness of the solution $(x(t), y(t))$ follows directly from the local Lipschitzian condition on $g$.

On the basis of Lemma 4, we can define the Poincaré map $P:(-1,+\infty) \times \mathbf{R} \rightarrow \mathbf{R}^{2}$ as follows:

$$
P:\left(x_{0}, y_{0}\right) \longrightarrow\left(x_{1}, y_{1}\right)=\left(x\left(2 \pi, x_{0}, y_{0}\right), y\left(2 \pi, x_{0}, y_{0}\right)\right) \text {. }
$$

We know that fixed points of the Poincaré map $P$ correspond to $2 \pi$-periodic solutions of (13).

To show the position of orbit $(x(t), y(t))$ of (13), we introduce a function $\zeta:(-1,+\infty) \times \mathbf{R} \rightarrow \mathbf{R}^{+}$,

$$
\zeta(x, y)=x^{2}+y^{2}+\frac{1}{(1+x)^{2}} .
$$

Lemma 5. There exists a constant $c_{0}>0$ such that, for any $c \geq c_{0}, \Gamma_{c}: \zeta(x, y)=c$ is a closed star-shaped curve around the origin.

Proof. Consider autonomous system:

$$
x^{\prime}=y, \quad y^{\prime}=-x+\frac{1}{(1+x)^{3}} .
$$

Obviously, $\Gamma_{c}$ is one orbit of the autonomous system above. From the expression of $\zeta$ we know that there exists $c_{1}>0$ such that, for $c \geq c_{1}, \Gamma_{c}$ is a closed curve around the origin. Applying the polar coordinate transformation $x=\rho \cos \vartheta$, $y=\rho \sin \vartheta$ to this system, we get

$$
\vartheta^{\prime}(t)=-1+\frac{\cos \vartheta}{\rho(1+\rho \cos \vartheta)^{3}} .
$$

In the case when $-1<\rho \cos \vartheta \leq 0$, we have $\vartheta^{\prime}(t) \leq-1$. In the case when $\cos \vartheta \geq 0$ and $\rho \geq 2$, we have $\cos \vartheta /(\rho(1+$ $\left.\rho \cos \vartheta)^{3}\right) \leq 1 / 2$, which implies $\vartheta^{\prime}(t) \leq-1 / 2$. Therefore, there exists $c_{2}>0$ such that, for $\zeta(\rho \cos \vartheta, \rho \sin \vartheta) \geq c_{2}, \vartheta(t)$ is decreasing strictly. Take $c_{0}=\max \left\{c_{1}, c_{2}\right\}$. Then for $c \geq c_{0}$, $\Gamma_{c}$ is a closed star-shaped curve around the origin.

Lemma 6 (see [1]). Assume that conditions $\left(h_{1}^{\prime}\right),\left(h_{2}^{\prime}\right)$, and $\left(h_{3}\right)$ hold. Then, for any $T>0$ and $\varrho>0$, there exists $\varrho_{0}>0$ sufficiently large such that, for $\zeta\left(x_{0}, y_{0}\right) \geq \varrho_{0}^{2}$,

$$
\zeta(x(t), y(t)) \geq \varrho^{2}, \quad t \in[0, T],
$$

where $(x(t), y(t))$ is the solution of (13) through the initial point $\left(x_{0}, y_{0}\right)$.

From Lemma 6 we know that if $\zeta\left(x_{0}, y_{0}\right)$ is large enough, then $x^{2}(t)+y^{2}(t)>0, t \in[0, T]$. Therefore, we can take the polar coordinate transformation

$$
x=r \cos \theta, \quad y=r \sin \theta .
$$

Under this transformation, system (13) becomes

$$
\begin{aligned}
& \frac{d r}{d t}=r \sin \theta \cos \theta-g(r \cos \theta) \sin \theta+p(t) \sin \theta, \\
& \frac{d \theta}{d t}=-\sin ^{2} \theta-\frac{1}{r} g(r \cos \theta) \cos \theta+\frac{1}{r} p(t) \cos \theta .
\end{aligned}
$$

Let $(r(t), \theta(t))=\left(r\left(t, r_{0}, \theta_{0}\right), \theta\left(t, r_{0}, \theta_{0}\right)\right)$ be the solution of (27) satisfying condition

$$
r(0)=r_{0}, \quad \theta(0)=\theta_{0}
$$

with $x_{0}=r_{0} \cos \theta_{0}, y_{0}=r_{0} \sin \theta_{0}$.

Then we can rewrite the Poincare map $P$ as follows:

$$
P:\left(r_{0}, \theta_{0}\right) \longrightarrow\left(r_{1}, \theta_{1}\right)=\left(r\left(2 \pi, r_{0}, \theta_{0}\right), \theta\left(2 \pi, r_{0}, \theta_{0}\right)\right),
$$

with $x_{0}=r_{0} \cos \theta_{0}>-1, y_{0}=r_{0} \sin \theta_{0}$.

Lemma 7. Assume that conditions $\left(h_{1}^{\prime}\right),\left(h_{2}^{\prime}\right)$, and $\left(h_{3}\right)$ hold. Then, for any $T>0$, there exist $\rho_{0}>0$ and $\omega>0$ such that, for $\zeta\left(x_{0}, y_{0}\right) \geq \rho_{0}^{2}$,

$$
\theta^{\prime}(t) \leq-\omega, \quad t \in[0, T]
$$

Proof. From $\left(h_{3}\right)$ we know that there exist constants $\alpha>0$ and $c>0$ such that

$$
\frac{g(x)-p(t)}{x} \geq \alpha, \quad x \in(c,+\infty), t \in \mathbf{R} .
$$

Moreover, we know from $\left(h_{1}^{\prime}\right)$ that there exist $\beta>0$ and $-1<$ $d<0$ such that

$$
\frac{g(x)-p(t)}{x} \geq \beta, \quad x \in(-1, d), t \in \mathbf{R} .
$$


If $x(t)>c, t \in[0, T]$, then

$$
\theta^{\prime}(t) \leq-\sin ^{2} \theta-\alpha \cos ^{2} \theta \leq-\min (1, \alpha) .
$$

If $-1<x(t)<d<0, t \in[0, T]$, then

$$
\theta^{\prime}(t) \leq-\sin ^{2} \theta-\beta \cos ^{2} \theta \leq-\min (1, \beta) .
$$

On the other hand, we know from Lemma 6 that there exists $\rho_{0}>0$ large enough such that if $\zeta\left(x_{0}, y_{0}\right) \geq \rho_{0}^{2}$ and $x(t) \in$ $[d, c], t \in[0, T]$, then

$$
\frac{|g(x(t))-p(t)|}{r(t)} \leq \frac{1}{3}, \quad|\sin \theta(t)| \geq \frac{\sqrt{2}}{2} .
$$

Hence,

$$
\theta^{\prime}(t) \leq-\sin ^{2} \theta(t)+\frac{|g(x(t))-p(t)|}{r(t)}|\cos \theta(t)| \leq-\frac{1}{6} .
$$

Consequently, the conclusion of Lemma 7 holds.

Lemma 8. Assume that conditions $\left(h_{1}^{\prime}\right),\left(h_{2}^{\prime}\right),\left(h_{3}\right)$, and $(\tau)$ hold. Let $A \geq 0$ be a given constant. Then we have

$$
\begin{aligned}
& \lim _{c \rightarrow+\infty} \int_{0}^{c} \frac{d s}{\sqrt{G(c)-G(s)-A(c-s)}}=0, \\
& \lim _{c \rightarrow-1^{+}} \int_{c}^{0} \frac{d s}{\sqrt{G(c)-G(s)+A(c-s)}}=0 .
\end{aligned}
$$

Proof. We now prove the first estimation. From condition $\left(h_{3}\right)$ we know that there exists a constant $\eta>1$ such that, for $\eta \leq s \leq c$,

$$
G(c)-G(s) \geq 2 A(c-s) .
$$

Then, for $\eta \leq s \leq c$, we have

$$
G(c)-G(s)-A(c-s) \geq \frac{1}{2}[G(c)-G(s)] .
$$

Write

$$
\int_{0}^{c} \frac{d s}{\sqrt{G(c)-G(s)-A(c-s)}}=I_{1}+I_{2}
$$

where

$$
\begin{aligned}
& I_{1}=\int_{0}^{\eta} \frac{d s}{\sqrt{G(c)-G(s)-A(c-s)}}, \\
& I_{2}=\int_{\eta}^{c} \frac{d s}{\sqrt{G(c)-G(s)-A(c-s)}} .
\end{aligned}
$$

From condition $\left(h_{3}\right)$ we can derive easily that $\lim _{c \rightarrow+\infty} I_{1}=0$. From (39) we get

$$
I_{2} \leq \sqrt{2} \int_{\eta}^{c} \frac{d s}{\sqrt{G(c)-G(s)}} \leq \sqrt{2} \int_{1}^{c} \frac{d s}{\sqrt{G(c)-G(s)}} .
$$

According to condition $(\tau)$, we have that $\lim _{c \rightarrow+\infty} I_{2}=0$. Hence, we get

$$
\lim _{c \rightarrow+\infty} \int_{0}^{c} \frac{d s}{\sqrt{G(c)-G(s)-A(c-s)}}=0 .
$$

Next, we prove the second estimation. Let $0<\varepsilon<1$ be a sufficiently small constant. In the case when $-1<c<-1+\varepsilon$, we write

$$
\int_{c}^{0} \frac{d s}{\sqrt{G(c)-G(s)+A(c-s)}}=J_{1}+J_{2},
$$

where

$$
\begin{aligned}
& J_{1}=\int_{c}^{-1+\varepsilon} \frac{d s}{\sqrt{G(c)-G(s)+A(c-s)}} \\
& J_{2}=\int_{-1+\varepsilon}^{0} \frac{d s}{\sqrt{G(c)-G(s)+A(c-s)}} .
\end{aligned}
$$

If $s \in(c,-1+\varepsilon) \subset(-1,-1+\varepsilon)$, then we have

$$
\begin{gathered}
G(c)-G(s)=g(\zeta)(c-s), \\
\zeta \in(c, s) \subset(-1,-1+\varepsilon) .
\end{gathered}
$$

Set

$$
\xi(\varepsilon)=\sup \{g(x): x \in(-1,-1+\varepsilon)\} .
$$

Obviously, $g(\zeta) \leq \xi(\varepsilon)$. From condition $\left(h_{1}^{\prime}\right)$ we know

$$
\lim _{\varepsilon \rightarrow 0^{+}} \xi(\varepsilon)=-\infty
$$

According to (46), we get that, for $s \in(c,-1+\varepsilon) \subset(-1,-1+\varepsilon)$,

$$
G(c)-G(s)=g(\zeta)(c-s) \geq \xi(\varepsilon)(c-s) .
$$

Hence,

$$
J_{1} \leq \int_{c}^{-1+\varepsilon} \frac{d s}{\sqrt{\xi(\varepsilon)(c-s)+A(c-s)}}=\frac{2 \sqrt{-1+\varepsilon-c}}{\sqrt{-\xi(\varepsilon)-A}}
$$

which, together with (48), means that $\lim _{c \rightarrow-1^{+}} J_{1}=0$. On the other hand, we can infer easily from $\left(h_{1}^{\prime}\right)$ that $\lim _{c \rightarrow-1^{+}} J_{2}=0$. Consequently, we have

$$
\lim _{c \rightarrow-1^{+}} \int_{c}^{0} \frac{d s}{\sqrt{G(c)-G(s)+A(c-s)}}=0 .
$$

Thus, the proof is completed.

Lemma 9. Assume that conditions $\left(h_{1}^{\prime}\right),\left(h_{2}^{\prime}\right),\left(h_{3}\right)$, and $(\tau)$ hold. Let $m$ be a given positive integer. Then, for any given positive integer $n$, there is a constant $R_{n}>0$ such that, for $\zeta\left(x_{0}, y_{0}\right) \geq R_{n}^{2}$,

$$
\theta(2 m \pi)-\theta_{0}<-2 n \pi .
$$


Proof. From Lemmas 6 and 7 we know that, for any sufficiently large $\varrho>0$, there is a constant $\varrho_{0}>\varrho$ such that, for $\zeta\left(x_{0}, y_{0}\right) \geq \varrho_{0}^{2}$ and $t \in[0,2 m \pi]$,

$$
\begin{gathered}
\zeta(x(t), y(t)) \geq \varrho^{2}, \\
\theta^{\prime}(t)<0 .
\end{gathered}
$$

Let $(x(t), y(t))$ be a solution of (13) satisfying $\zeta\left(x_{0}, y_{0}\right) \geq \varrho_{0}^{2}$. Then the solution $(x(t), y(t))$ will move clockwise during the time period $[0,2 m \pi]$. Without loss of generality, we assume that $\left(x_{0}, y_{0}\right)$ lies in the first quadrant. Then there exist $t_{0}=$ $0<t_{1}<t_{2}<t_{3}<t_{4}<t_{5}$ such that

$$
\begin{aligned}
& x\left(t_{1}\right)>0, \quad y\left(t_{1}\right)=0 ; \quad x(t)>0, \quad y(t)>0, \\
& t \in\left(t_{0}, t_{1}\right), \\
& x\left(t_{2}\right)=0, \quad y\left(t_{2}\right)<0 ; \quad x(t)>0, \quad y(t)<0, \\
& t \in\left(t_{1}, t_{2}\right) \text {, } \\
& x\left(t_{3}\right)<0, \quad y\left(t_{3}\right)=0 ; \quad x(t)<0, \quad y(t)<0, \\
& t \in\left(t_{2}, t_{3}\right) \text {, } \\
& x\left(t_{4}\right)=0, \quad y\left(t_{4}\right)>0 ; \quad x(t)<0, \quad y(t)>0, \\
& t \in\left(t_{3}, t_{4}\right) \text {, } \\
& x\left(t_{5}\right)>0, \quad y\left(t_{5}\right)=0 ; \quad x(t)>0, \quad y(t)>0, \\
& t \in\left(t_{4}, t_{5}\right) \text {. }
\end{aligned}
$$

Next, we will estimate $t_{i}-t_{i-1}(i=1,2,3,4,5)$, respectively. We first estimate $t_{1}-t_{0}$. If $t \in\left[t_{0}, t_{1}\right]$, then $y(t) \geq 0$. Let us define an auxiliary function

$$
w(t)=\frac{1}{2} y^{2}(t)+G(x(t))-M x(t),
$$

where $M=\max \{|p(t)|: t \in[0,2 \pi]\}$. Then we have that, for $t \in\left[t_{0}, t_{1}\right]$,

$$
\begin{aligned}
w^{\prime}(t) & =y(t) y^{\prime}(t)+g(x(t)) x^{\prime}(t)-M x^{\prime}(t) \\
& =y(t)(p(t)-M) \leq 0,
\end{aligned}
$$

which implies that $w(t)$ is decreasing on the interval $\left[t_{0}, t_{1}\right]$. Therefore, we get that, for $t \in\left[t_{0}, t_{1}\right]$,

$$
\frac{1}{2} y^{2}(t)+G(x(t))-M x(t) \geq G\left(x\left(t_{1}\right)\right)-M x\left(t_{1}\right),
$$

which means

$$
x^{\prime}(t) \geq \sqrt{2\left(G\left(x\left(t_{1}\right)\right)-G(x(t))\right)-2 M\left(x\left(t_{1}\right)-x(t)\right)} .
$$

Hence, we obtain

$$
\begin{aligned}
t_{1} & -t_{0} \\
& \leq \int_{x\left(t_{0}\right)}^{x\left(t_{1}\right)} \frac{d x}{\sqrt{2\left(G\left(x\left(t_{1}\right)\right)-G(x)\right)-2 M\left(x\left(t_{1}\right)-x\right)}} \\
& \leq \int_{0}^{x\left(t_{1}\right)} \frac{d x}{\sqrt{2\left(G\left(x\left(t_{1}\right)\right)-G(x)\right)-2 M\left(x\left(t_{1}\right)-x\right)}} .
\end{aligned}
$$

Similarly, we can obtain

$$
t_{5}-t_{4} \leq \int_{0}^{x\left(t_{5}\right)} \frac{d x}{\sqrt{2\left(G\left(x\left(t_{5}\right)\right)-G(x)\right)-2 M\left(x\left(t_{5}\right)-x\right)}} .
$$

We next estimate $t_{2}-t_{1}$. If $t \in\left[t_{1}, t_{2}\right]$, then $y(t) \leq 0$. Therefore, we have

$$
w^{\prime}(t)=y(t)(p(t)-M) \geq 0, \quad t \in\left[t_{1}, t_{2}\right],
$$

which implies that $w(t)$ is increasing on the interval $\left[t_{1}, t_{2}\right]$. Furthermore, we have that, for $t \in\left[t_{1}, t_{2}\right]$,

$$
\frac{1}{2} y^{2}(t)+G(x(t))-M x(t) \geq G\left(x\left(t_{1}\right)\right)-M x\left(t_{1}\right),
$$

which yields

$$
-x^{\prime}(t) \geq \sqrt{2\left(G\left(x\left(t_{1}\right)\right)-G(x(t))\right)-2 M\left(x\left(t_{1}\right)-x(t)\right)} .
$$

Consequently, we get

$$
t_{2}-t_{1} \leq \int_{0}^{x\left(t_{1}\right)} \frac{d x}{\sqrt{2\left(G\left(x\left(t_{1}\right)\right)-G(x)\right)-2 M\left(x\left(t_{1}\right)-x\right)}} .
$$

We now estimate $t_{3}-t_{2}$. If $t \in\left[t_{2}, t_{3}\right]$, then $y(t) \leq 0$. Define

$$
\widetilde{w}(t)=\frac{1}{2} y^{2}(t)+G(x(t))+M x(t) .
$$

Then we have that, for $t \in\left[t_{2}, t_{3}\right]$,

$$
\widetilde{w}(t)=y(t)(p(t)+M) \leq 0,
$$

which implies that $\widetilde{w}(t)$ is decreasing on the interval $\left[t_{2}, t_{3}\right]$. Therefore, we get that, for $t \in\left[t_{2}, t_{3}\right]$,

$$
\frac{1}{2} y^{2}(t)+G(x(t))+M x(t) \geq G\left(x\left(t_{3}\right)\right)+M x\left(t_{3}\right),
$$

which implies

$$
-x^{\prime}(t) \geq \sqrt{2\left(G\left(x\left(t_{3}\right)\right)-G(x(t))\right)+2 M\left(x\left(t_{3}\right)-x(t)\right)} .
$$

Hence,

$$
t_{3}-t_{2} \leq \int_{x\left(t_{3}\right)}^{0} \frac{d x}{\sqrt{2\left(G\left(x\left(t_{3}\right)\right)-G(x)\right)+2 M\left(x\left(t_{3}\right)-x\right)}} .
$$

Similarly, we get

$$
t_{4}-t_{3} \leq \int_{x\left(t_{3}\right)}^{0} \frac{d x}{\sqrt{2\left(G\left(x\left(t_{3}\right)\right)-G(x)\right)+2 M\left(x\left(t_{3}\right)-x\right)}} .
$$


According to Lemma 6, if we take $\varrho_{0}>>1$, then we have $x\left(t_{1}\right)>>1,0<1+x\left(t_{3}\right)<<1$ and $x\left(t_{5}\right)>>1$. From Lemma 8 and (59)-(70) we know that, for any sufficiently small $\varepsilon>0$, there exists $\varrho_{0}>>1$ such that

$$
t_{i}-t_{i-1}<\varepsilon, \quad(i=1,2,3,4,5) .
$$

It follows that

$$
t_{5}-t_{0}<5 \varepsilon
$$

Therefore, the motion $(x(t), y(t))$ rotates clockwise a turn in a period less than $5 \varepsilon$. Consequently, $(x(t), y(t))$ can rotate a sufficiently large number of turns during the period $2 m \pi$ provided that $\zeta\left(x_{0}, y_{0}\right) \geq \varrho_{0}^{2}\left(\varrho_{0}>>1\right)$ is satisfied.

The proof is thus completed.

\section{Infinity of Harmonic Solutions}

To prove Theorem 1, we first prove the following proposition.

Proposition 10. Assume that conditions $\left(h_{1}^{\prime}\right),\left(h_{2}^{\prime}\right),\left(h_{3}\right)$, and $(\tau)$ hold. Then $\left(1^{\prime}\right)$ has infinitely many harmonic solutions $\left\{x_{j}(t)\right\}$ satisfying

$$
\begin{gathered}
\lim _{j \rightarrow \infty}\left(\min _{0 \leq t \leq 2 \pi}\left(1+x_{j}(t)+\left|x_{j}^{\prime}(t)\right|\right)\right)=0, \\
\lim _{j \rightarrow \infty}\left(\max _{0 \leq t \leq 2 \pi}\left(1+x_{j}(t)+\left|x_{j}^{\prime}(t)\right|\right)\right)=+\infty .
\end{gathered}
$$

Proof. From Lemma 6 we know that there exist $a_{1}>c_{0}\left(c_{0}\right.$ is given in Lemma 5) and $\omega_{1}>0$ such that, for $\zeta\left(x_{0}, y_{0}\right) \geq a_{1}^{2}$, $\zeta(x(t), y(t)) \geq 2$ and $\theta^{\prime}(t)<-\omega_{1}, t \in[0,2 \pi]$. For $\zeta\left(x_{0}, y_{0}\right) \geq$ $a_{1}^{2}$, we consider

$$
\Phi\left(r_{0}, \theta_{0}\right)=\theta\left(2 \pi, r_{0}, \theta_{0}\right)-\theta_{0}
$$

with $x_{0}=r_{0} \cos \theta_{0}, y_{0}=r_{0} \sin \theta_{0}$. Obviously, there exists an integer $k \geq 1$ such that

$$
\theta\left(2 \pi, r_{0}, \theta_{0}\right)-\theta_{0}>-2 k \pi, \quad \text { for } \zeta\left(r_{0} \cos \theta_{0}, r_{0} \sin \theta_{0}\right)=a_{1}^{2} \text {. }
$$

On the other hand, it follows from Lemma 9 that there exists $a_{2}>a_{1}$ such that

$$
\theta\left(2 \pi, r_{0}, \theta_{0}\right)-\theta_{0}<-2 k \pi, \quad \text { for } \zeta\left(r_{0} \cos \theta_{0}, r_{0} \sin \theta_{0}\right)=a_{2}^{2} \text {. }
$$

Meanwhile, there exists an integer $k^{\prime}>k$ such that

$\theta\left(2 \pi, r_{0}, \theta_{0}\right)-\theta_{0}>-2 k^{\prime} \pi, \quad$ for $\zeta\left(r_{0} \cos \theta_{0}, r_{0} \sin \theta_{0}\right)=a_{2}^{2}$.

From (75) and (76) we know that the area-preserving homeomorphism $P$ is twisting on the annulus $A_{1}=\{(x, y) \epsilon$ $\left.(-1,+\infty) \times \mathbf{R}: a_{1} \leq \zeta(x, y) \leq a_{2}\right\}$. Obviously, we have $r(2 \pi)>$ 0 provided that $\zeta\left(x_{0}, y_{0}\right) \geq a_{1}^{2}$. Hence, $O \in P(D)$, where $D$ is an open region with boundary $\zeta(x, y)=a_{1}^{2}$. Finally, we know from Lemma 5 that both $\Gamma_{a_{1}}: \zeta(x, y)=a_{1}^{2}$ and $\Gamma_{a_{2}}: \zeta(x, y)=a_{2}^{2}$ are closed star-shaped curves with respect to the origin $O$. Thus, we have proved that all conditions of the generalized Poincaré-Birkhoff theorem [16, 17] are satisfied. Consequently, the Poincaré map $P$ has at least two fixed points $\left(r_{1 i}, \theta_{1 i}\right)(i=1,2)$ in annulus $A_{1}$ and then (13) has two $2 \pi$ periodic solutions $\left(x_{1 i}(t), y_{1 i}(t)\right)=$ $\left(x\left(t, x_{1 i}, y_{1 i}\right), y\left(t, x_{1 i}, y_{1 i}\right)\right)\left(x_{1 i}=r_{1 i} \cos \theta_{1 i}, y_{1 i}=r_{1 i} \sin \theta_{1 i}\right)$. Therefore, $x_{1 i}(t)$ are $2 \pi$ periodic solutions of $\left(1^{\prime}\right)$. On the other hand, since the period of any periodic solution of $\left(1^{\prime}\right)$ must be multiple of the period of $p(t)$, then $2 \pi$ is the minimal period of $x_{1 i}(t)$. Therefore, $x_{1 i}(t)$ are harmonic solutions of $\left(1^{\prime}\right)$.

Similarly, we can find a sequence

$$
\left(a_{1}<a_{2}<\right) a_{3}<\cdots<a_{j}<a_{j+1}<\cdots, \quad \lim _{j \rightarrow \infty} a_{j}=+\infty,
$$

such that the area-preserving homeomorphism $P$ is twisting on the annuli

$$
\begin{array}{r}
A_{j}=\left\{(x, y) \in(-1,+\infty) \times \mathbf{R}: a_{j} \leq \zeta(x, y) \leq a_{j+1}\right\} \\
j=2,3, \ldots
\end{array}
$$

Therefore, the Poincaré map $P$ has at least two fixed points $\left(r_{j i}, \theta_{j i}\right)(i=1,2)$ in each $A_{j},(j=2,3, \ldots)$. Consequently, (13) has two $2 \pi$ periodic solutions $\left(x_{j i}(t), y_{j i}(t)\right)=$ $\left(x\left(t, x_{j i}, y_{j i}\right), y\left(t, x_{j i}, y_{j i}\right)\right)\left(x_{j i}=r_{j i} \cos \theta_{j i}, y_{j i}=r_{j i} \sin \theta_{j i}\right)$ and then $x_{j i}(t)$ are $2 \pi$ periodic solutions of $\left(1^{\prime}\right)$. Similarly, we know that $x_{j i}(t)$ are harmonic solutions of $\left(1^{\prime}\right)$. Since $\lim _{j \rightarrow \infty} a_{j}=+\infty$, we have

$$
\begin{gathered}
\min \left\{x+1+|y|: \zeta(x, y)=a_{j}\right\} \rightarrow 0, \quad j \longrightarrow \infty \\
\max \left\{x+1+|y|: \zeta(x, y)=a_{j}\right\} \longrightarrow+\infty, \quad j \longrightarrow \infty .
\end{gathered}
$$

Furthermore, we know from Lemma 6 that, for $i=1,2$,

$$
\begin{aligned}
& \lim _{j \rightarrow \infty}\left(\min _{0 \leq t \leq 2 \pi}\left(1+x_{j i}(t)+\left|x_{j i}^{\prime}(t)\right|\right)\right)=0, \\
& \lim _{j \rightarrow \infty}\left(\max _{0 \leq t \leq 2 \pi}\left(1+x_{j i}(t)+\left|x_{j i}^{\prime}(t)\right|\right)\right)=+\infty .
\end{aligned}
$$

Thus we have proved Proposition 10.

Proof of Theorem 1. Consider the equivalent equation of (1):

$$
u^{\prime \prime}+\widetilde{g}(u)=p(t)
$$

where $\tilde{g}(u)=g(1+u)$. Obviously, $\tilde{g}$ satisfies conditions $\left(h_{1}^{\prime}\right)$, $\left(h_{2}^{\prime}\right)$, and $\left(h_{3}\right)$. To use Proposition 10 , we only need to prove that condition $(\tau)$ holds for function $\widetilde{G}(u)\left(=\int_{1}^{u} \widetilde{g}(s) d s\right)$. Set

$$
\tilde{\tau}(c)=\int_{1}^{c} \frac{d s}{\sqrt{\widetilde{G}(c)-\widetilde{G}(s)}} .
$$


Then we have

$$
\tilde{\tau}(c)=\int_{1}^{1+c} \frac{d s}{\sqrt{G(1+c)-G(s)}}-\int_{1}^{2} \frac{d s}{\sqrt{G(1+c)-G(s)}} .
$$

From conditions $(\tau)$ and $\left(h_{3}\right)$ we get $\lim _{c \rightarrow+\infty} \tilde{\tau}(c)=0$. Therefore, all conditions of Proposition 10 are satisfied. Accordingly, (82) has infinitely many harmonic solutions $\left\{u_{j}(t)\right\}$ satisfying

$$
\begin{gathered}
\lim _{j \rightarrow \infty}\left(\min _{0 \leq t \leq 2 \pi}\left(1+u_{j}(t)+\left|u_{j}^{\prime}(t)\right|\right)\right)=0, \\
\lim _{j \rightarrow \infty}\left(\max _{0 \leq t \leq 2 \pi}\left(1+u_{j}(t)+\left|u_{j}^{\prime}(t)\right|\right)\right)=+\infty .
\end{gathered}
$$

Recalling that (82) is obtained by taking a parallel transformation $x=1+u$ to (1), we know that the conclusion of Theorem 1 holds.

Remark 11. In [16], the Poincaré-Birkhoff theorem was proved in case that the inner closed curve of the annulus is star shaped. From [17] we know that there is a need for both boundaries of the annulus to be star shaped in the PoincaréBirkhoff theorem.

\section{Infinity of Subharmonic Solutions}

To prove Theorem 2, we first prove the following proposition.

Proposition 12. Assume that conditions $\left(h_{1}^{\prime}\right),\left(h_{2}^{\prime}\right),\left(h_{3}\right)$, and $(\tau)$ hold. Then, for any given integer $m \geq 2,\left(1^{\prime}\right)$ has infinitely many $m$-order subharmonic solutions $\left\{x_{j}(t)\right\}$ satisfying

$$
\begin{gathered}
\lim _{j \rightarrow \infty}\left(\min _{0 \leq t \leq 2 m \pi}\left(1+x_{j}(t)+\left|x_{j}^{\prime}(t)\right|\right)\right)=0, \\
\lim _{j \rightarrow \infty}\left(\max _{0 \leq t \leq 2 m \pi}\left(1+x_{j}(t)+\left|x_{j}^{\prime}(t)\right|\right)\right)=+\infty .
\end{gathered}
$$

Proof. Let $m \geq 2$ be a given integer. From Lemmas 6 and 9 we know that there exists $b_{1}>c_{0}\left(c_{0}\right.$ is given in Lemma 5$)$ and $\omega_{1}^{\prime}>0$ such that, for $\zeta\left(x_{0}, y_{0}\right) \geq b_{1}^{2}$,

$$
\begin{gathered}
\theta^{\prime}(t)<-\omega_{1}^{\prime}, \quad \zeta(x(t), y(t)) \geq 2, \quad t \in[0,2 m \pi], \\
\theta\left(2 \pi, r_{0}, \theta_{0}\right)-\theta_{0}<-2 \pi .
\end{gathered}
$$

For $\zeta\left(x_{0}, y_{0}\right) \geq b_{1}^{2}$, we consider

$$
\Psi\left(r_{0}, \theta_{0}\right)=\theta\left(2 m \pi, r_{0}, \theta_{0}\right)-\theta_{0},
$$

with $x_{0}=r_{0} \cos \theta_{0}, y_{0}=r_{0} \sin \theta_{0}$. Obviously, there exists a positive prime integer $q$ such that

$$
\begin{array}{r}
\theta\left(2 m \pi, r_{0}, \theta_{0}\right)-\theta_{0}>-2 q \pi, \\
\text { for } \zeta\left(r_{0} \cos \theta_{0}, r_{0} \sin \theta_{0}\right)=b_{1}^{2} .
\end{array}
$$

On the other hand, it follows from Lemma 8 that there exists $b_{2}>b_{1}$ such that

$$
\begin{array}{r}
\theta\left(2 m \pi, r_{0}, \theta_{0}\right)-\theta_{0}<-2 q \pi, \\
\text { for } \zeta\left(r_{0} \cos \theta_{0}, r_{0} \sin \theta_{0}\right)=b_{2}^{2} .
\end{array}
$$

From (90) and (91) we know that the map $P^{m}$ is twisting on the annulus $B_{1}=\left\{(x, y) \in(-1,+\infty) \times \mathbf{R}: b_{1} \leq \zeta(x, y) \leq\right.$ $\left.b_{2}\right\}$. Using the generalized Poincaré-Birkhoff twist theorem, we know that $P^{m}$ has at least two fixed points $\left(r_{m 1 i}, \theta_{m 1 i}\right)(i=$ $1,2)$ in $B_{1}$, which satisfy

$$
\theta\left(2 m \pi, r_{m 1 i}, \theta_{m 1 i}\right)-\theta_{m 1 i}=-2 q \pi, \quad(i=1,2) .
$$

It follows that (13) has two $2 m \pi$-periodic solutions $\left(x_{m 1 i}(t)\right.$, $\left.y_{m 1 i}(t)\right)$ and then $\left(1^{\prime}\right)$ has two $2 m \pi$-periodic solutions $x_{m 1 i}(t)$.

Next, we will prove that $2 m \pi$ is the minimal period of $x_{m 1 i}(t)$. Assume by contradiction that $2 l \pi(1 \leq l \leq m-1)$ is the minimal period of $x_{m 1 i}(t)$. Then we have $m=n l(n \geq 2)$. Since $\left(x_{m 1 i}(t), y_{m 1 i}(t)\right)$ is $2 l \pi$ periodic, we know from $(88)$ that there exists a positive integer $k_{i} \geq 2$ such that

$$
\theta\left(2 l \pi, r_{m 1 i}, \theta_{m 1 i}\right)-\theta_{m 1 i}=-2 k_{i} \pi \text {. }
$$

Furthermore,

$$
\theta\left(2 m \pi, r_{m 1 i}, \theta_{m 1 i}\right)-\theta_{m 1 i}=-2 n k_{i} \pi .
$$

Hence, we have $n k_{i}=q$. Since $q$ is a prime integer, we get a contradiction. This proves that $2 m \pi$ is the minimal period of $x_{m 1 i}(t)$. Consequently, $x_{m 1 i}(t)$ are $m$-order subharmonic solutions of $\left(1^{\prime}\right)$.

In a similar manner, we can find a sequence

$$
\left(b_{1}<b_{2}<\right) b_{3}<\cdots<b_{j}<b_{j+1}<\cdots, \quad \lim _{j \rightarrow \infty} b_{j}=+\infty,
$$

such that the area-preserving homeomorphism $P^{m}$ is twisting on the annuli

$$
B_{j}=\left\{(x, y) \in(-1,+\infty) \times \mathbf{R}: b_{j} \leq \zeta(x, y) \leq b_{j+1}\right\} .
$$

Therefore, the Poincaré map $P^{m}$ has at least two fixed points $\left(r_{m j i}, \theta_{m j i}\right)(i=1,2)$ in each $B_{j},(j=2,3, \ldots)$. Consequently, (13) has two $2 m \pi$ periodic solutions $\left(x_{m j i}(t), y_{m j i}(t)\right)$ and then $x_{m j i}(t)$ are $2 m \pi$ periodic solutions of $\left(1^{\prime}\right)$. Similarly, we know that $x_{m j i}(t)$ are $m$-order subharmonic solutions of $\left(1^{\prime}\right)$. Since $\lim _{j \rightarrow \infty} b_{j}=+\infty$, we have

$$
\begin{gathered}
\min \left\{x+1+|y|: \zeta(x, y)=b_{j}\right\} \longrightarrow 0, \quad j \longrightarrow \infty \\
\max \left\{x+1+|y|: \zeta(x, y)=b_{j}\right\} \longrightarrow+\infty, \quad j \longrightarrow \infty .
\end{gathered}
$$

Furthermore, we know from Lemma 6 that, for $i=1,2$,

$$
\begin{gathered}
\lim _{j \rightarrow \infty}\left(\min _{0 \leq t \leq 2 m \pi}\left(1+x_{m j i}(t)+\left|x_{m j i}^{\prime}(t)\right|\right)\right)=0, \\
\lim _{j \rightarrow \infty}\left(\max _{0 \leq t \leq 2 m \pi}\left(1+x_{m j i}(t)+\left|x_{m j i}^{\prime}(t)\right|\right)\right)=+\infty .
\end{gathered}
$$

Thus we have proved Proposition 12.

Proof of Theorem 2. Using Proposition 12 and the same method as proving Theorem 1, we can prove Theorem 2 . 


\section{Conflict of Interests}

The authors declare that there is no conflict of interests regarding the publication of this paper.

\section{Acknowledgments}

This research was supported by the Research Fund for the Doctoral Program of Higher Education of China, no. 11AA0013, and the Grant of Beijing Education Committee Key Project, no. KZ201310028031.

\section{References}

[1] M. A. del Pino and R. F. Manásevich, "Infinitely many T-periodic solutions for a problem arising in nonlinear elasticity," Journal of Differential Equations, vol. 103, no. 2, pp. 260-277, 1993.

[2] A. C. Lazer and S. Solimini, "On periodic solutions of nonlinear differential equations with singularities," Proceedings of the American Mathematical Society, vol. 99, no. 1, pp. 109-114, 1987.

[3] T. Ding, Applications of Qualitative Methods of Ordinary Differential Equations, Higher Education Press, Beijing, China, 2004.

[4] Z. Wang and T. Ma, "Existence and multiplicity of periodic solutions of semilinear resonant Duffing equations with singularities," Nonlinearity, vol. 25, no. 2, pp. 279-307, 2012.

[5] Z. Wang, "Periodic solutions of the second-order differential equations with singularity, Nonlinear Analysis, vol. 58, no. 3-4, pp. 319-331, 2004.

[6] A. Fonda, R. Manásevich, and F. Zanolin, "Subharmonic solutions for some second-order differential equations with singularities," SIAM Journal on Mathematical Analysis, vol. 24, no. 5, pp. 1294-1311, 1993.

[7] A. Fonda and M. Garrione, "A Landesman-Lazer-type condition for asymptotically linear second-order equations with a singularity," Proceedings of the Royal Society of Edinburgh A, vol. 142, no. 6, pp. 1263-1277, 2012.

[8] P. Habets and L. Sanchez, "Periodic solutions of some Liénard equations with singularities," Proceedings of the American Mathematical Society, vol. 109, no. 4, pp. 1035-1044, 1990.

[9] D. Jiang, J. Chu, and M. Zhang, "Multiplicity of positive periodic solutions to superlinear repulsive singular equations," Journal of Differential Equations, vol. 211, no. 2, pp. 282-302, 2005.

[10] X. Li and Z. Zhang, "Periodic solutions for second-order differential equations with a singular nonlinearity," Nonlinear Analysis, vol. 69, no. 11, pp. 3866-3876, 2008.

[11] I. Rachunková, M. Tvrdý, and I. Vrkoč, "Existence of nonnegative and nonpositive solutions for second order periodic boundary value problems," Journal of Differential Equations, vol. 176, no. 2, pp. 445-469, 2001.

[12] P. J. Torres, "Existence of one-signed periodic solutions of some second-order differential equations via a Krasnoselskii fixed point theorem," Journal of Differential Equations, vol. 190, no. 2, pp. 643-662, 2003.

[13] M. Zhang, "Periodic solutions of Liénard equations with singular forces of repulsive type," Journal of Mathematical Analysis and Applications, vol. 203, no. 1, pp. 254-269, 1996.

[14] J. Chu, P. J. Torres, and M. Zhang, "Periodic solutions of second order non-autonomous singular dynamical systems," Journal of Differential Equations, vol. 239, no. 1, pp. 196-212, 2007.
[15] J. Chu, N. Fan, and P. J. Torres, "Periodic solutions for second order singular damped differential equations," Journal of Mathematical Analysis and Applications, vol. 388, no. 2, pp. 665-675, 2012.

[16] W. Y. Ding, "A generalization of the Poincaré-Birkhoff theorem," Proceedings of the American Mathematical Society, vol. 88, no. 2, pp. 341-346, 1983.

[17] P. Le Calvez and J. Wang, "Some remarks on the Poincaré-Birkhoff theorem," Proceedings of the American Mathematical Society, vol. 138, no. 2, pp. 703-715, 2010. 


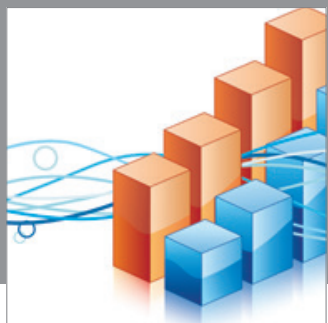

Advances in

Operations Research

mansans

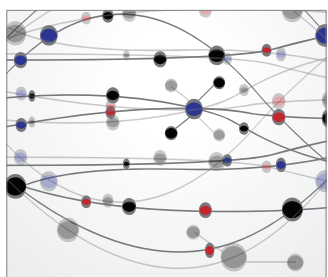

The Scientific World Journal
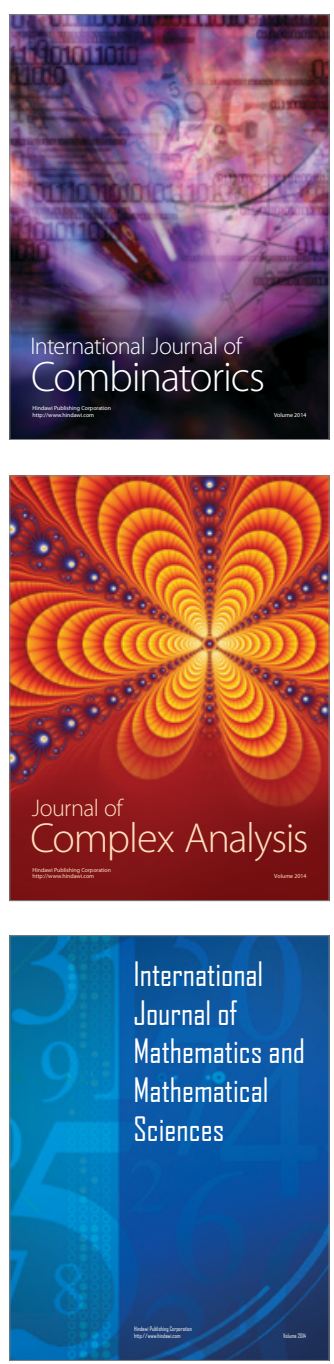
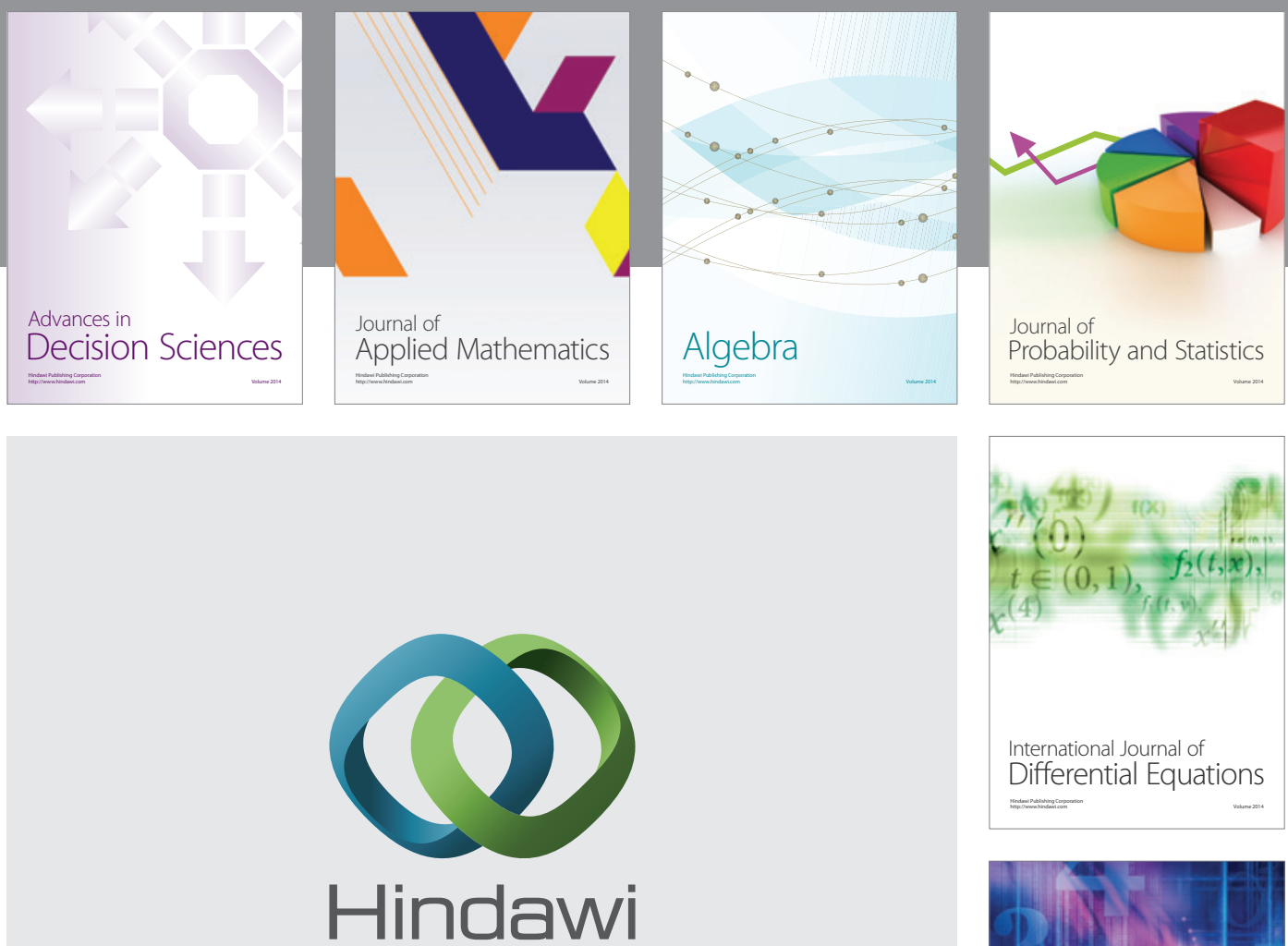

Submit your manuscripts at http://www.hindawi.com
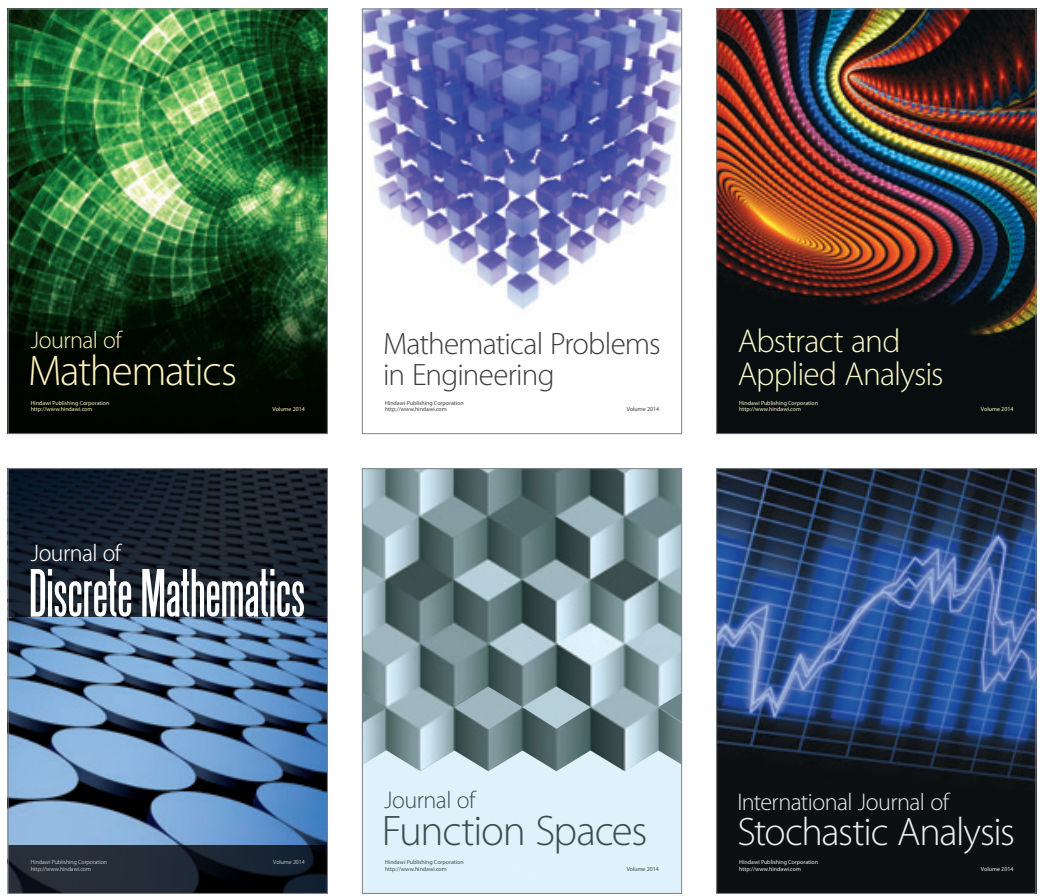

Journal of

Function Spaces

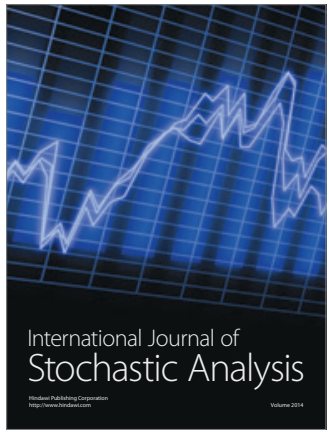

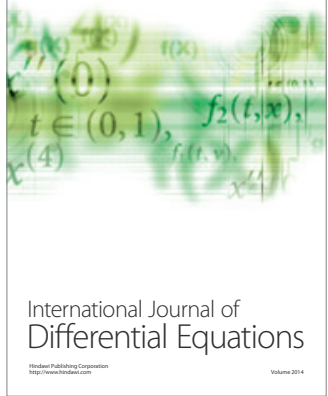
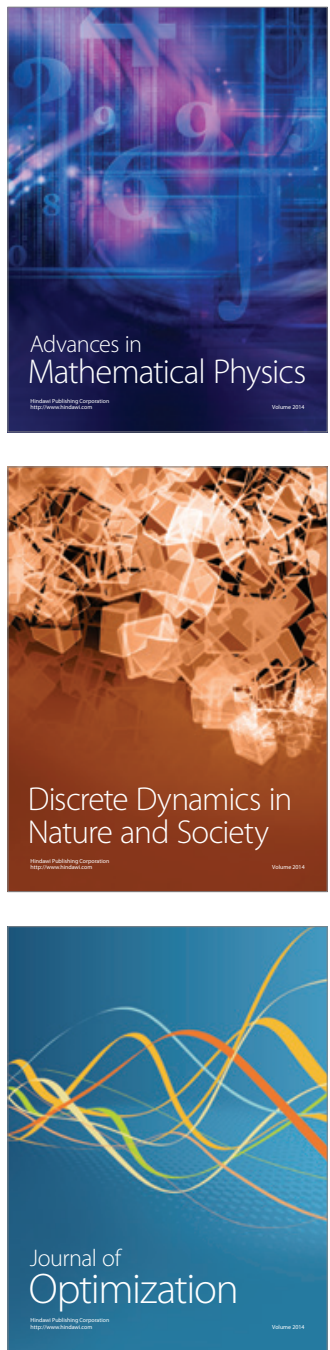